\title{
ZN-Face: A system for access control using automated face recognition
}

\author{
Jörg Kopecz, Wolfgang Konen and Ekkehard Schulze-Krüger \\ Zentrum für Neuroinformatik GmbH, Bochum \\ Universitätsstr. 160 \\ D-44801 Bochum \\ Germany
}

March 11, 2008

\begin{abstract}
We present a biometric access control device which is based on the identification of human faces. The system combines a console for semiautomated image acquisition with the necessary algorithms for face recognition. Facial features are stored in a relatively compact data format (1.6 kB). ZN-Face runs on a Pentium 90 without any special accelerator hardware where it performs image acquisition, face localization and identification in less than 3 seconds. ZN-Face not only allows robust identification of stored persons (despite changes in facial expression or size), but also reliable rejection of unknown persons. With an acceptance criterion which safely rejects all unknown persons we achieve an identification rate above $99 \%(F R R<1 \%)$. The $Z N$ Bochum $G m b H$ has sold more than 100 licences to various institutions and companies, among them the Kremlin in Moscow. The $Z N$ Bochum GmbH holds the relevant patents for $Z N-F a c e$.
\end{abstract}

\section{Introduction}

Face recognition is a remarkable example for the ability of humans to perform with great reliability a complex visual recognition task. We are able to recognize thousands of faces which we have learned during our lifetime. Our visual performance is very robust against a variety of factors like changes in facial expression, head posture or size, illumination, background, facial aging or partial occlusion of a face. It is one of the challenges of artificial information processing to achieve at least partially a similar performance on systems for automated visual recognition. The ease with which the problem is solved by the human brain, i. e. a biological neural system, may serve as a guideline in the sense that neural information processing paradigms can be important also for the construction of automated face recognition systems. 
In this contribution we will report on the development of the face recognition system ZN-Face which offers a turn-key solution for an access control system. ZN-Face is based on the neural algorithms of [1] which are briefly reviewed in Section 2 (Elastic Graph Matching). In Section 3 we describe the extensions necessary to migrate the algorithm into an automated access control system.

Numerous approaches for solving the face recognition problem can be found in the literature from which we can mention here only a few. A good and more comprehensive survey of the state of the art can be found in [2]. Early algorithms [3] use feature-based techniques (e. g. features like localization or thickness of eyebrows) or template matching for recognition [4]. Recently, a systematic comparision of feature-based vs. template-based algorithms has

been undertaken by Brunelli and Poggio [5]. Gilbert and Yang [6] presented a real-time face recognition system using custom VLSI hardware. The hardware allows fast template correlation and the system is able to perform an identification from a 34-person database in 2 to 3 seconds. Another approach uses the decomposition of facial images into an 'eigenface' expansion (similar to Karhune-Loeve expansion) [7,8]. These expansions achieve a very compact representation of faces, they are, however, rather sensitive against variations in imaging conditions.

Another group of algorithms (including this work) uses neural processing techniques for face recognition [9, 10] or gender recognition [11]. One of the few systems which does not only present a face recognition algorithm but also a complete access control booth has been proposed by Bichsel and Seitz [12]. This direction is also the aim of the current work, where a complete hardwaresetup has been developed as a biometric access control device for real-world security applications.

\section{Face recognition by graph matching}

The basic recognition algorithm of ZN-Face is an extension of the Elastic Graph Matching Algorithm [1]. In contrast to most other face recognition algorithms which require distinct processing steps like localization, separation, standardization and finally recognition of faces, the current algorithm is more coherent in the sense that one basic principle is used to perform the above steps simultaneously. Here, faces are stored as flexible graphs or grids (see Fig. 1) with characteristic visual features attached to the nodes of the graph (labeled graphs). A data representation with labeled graphs has computational advantages:

robustness: The features are invariant against intensity changes and contrast changes in the image. Furthermore, the features are less affected by changes in head posture, size and facial expression than raw grey level features.

data compression: compared to the raw image which has in our case a size of $128 \times 128$ pixels, the size of the graph (about $1.6 \mathrm{kB}$ ) is smaller by a factor of 10 . 
scaling: a sparse graph can be readily adjusted to changes in the geometry (size, perspective, see Fig. 1B,C). Change in size in a pixel-based data representation, for example, would require a more complicated transformation.

distribution: Compared to a high-level feature description (e.g. 'eye', 'nose') the graph contains sufficient information distribution on simple, but numerous features. Even if the information at a single graph node is missing due to occlusion, recognition is usually still possible due to the information at the remaining nodes.

In summary, the face recognition algorithm consists of three basic steps:

(1) Convolution of the image with feature extractors used in the labeled graph representation.

(2) A stored graph is matched to the image by an optimization procedure: Position, size and inner structure of the graph are varied in order to maximize the similarity between graph node features and corresponding image features. Penality terms inhibit too large deformations of the graph. As a result, a new graph with the actual image features as node labels can be extracted.

(3) In order to decide, whether stored graph and image show the face of the same person, the extracted graph is compared also to a number of reference graphs. Only if certain significance conditions are fulfilled the match is accepted.

\section{Access Control System ZN-Face}

ZN-Face offers an automated access control system which performs a biometric identification of persons from their facial images. To achieve this, several extensions are necessary to transform the Elastic Graph Matching algorithm of Sec. 2 into a system which is easy to use in real-world security applications:

- Fast computation on standard hardware: In former times the graph matching was performed on a transputer-based platform and required about 12 seconds for the identification. Now, the algorithm has been optimized and ported to a standard PC (Pentium PC/90 Mhz without special accelerator hardware). Computation time for a full identification is 3 seconds.

- Semi-automated image acquisition: No operator is available to adjust the camera (persons may differ in their size and their distance to the camera) and to trigger the image acquisition. An appropriate semi-automated procedure is described below. 
- Easy-to-use system administration: A Windows-based ${ }^{1}$ GUI has been developed which allows simple control by an authorized system administrator (e.g. adding or deleting access rights, monitoring the access protocol).

- Possibility to handle large databases (1000 persons and more).

In order to achieve the last point without unacceptable increase in computation time, we perform with ZN-Face verification instead of recognition. Verification has the additional advantage of higher security because in addition to a standard personal identification via secret number (PIN) or card, the face is used as independent control of the person's identity.

The basic verification procedure can be described as follows: The hardware setup of the system consists of a standard PC with framegrabber and a ZNFace console (a prototype is shown in Fig. 2) containing camera, ID acquisition device (card reader or PIN pad) and LC-display. The camera is positioned behind a semi-permeable mirror which is tilted about $30-50^{\circ}$ against the vertical line. Camera and mirror are tilted in order to allow users of different height to view and position themselves centrally in front of the camera. (at the expense of some size variation in the acquired facial images). The user triggers the image acquisition and identifies himself (PIN pad or card). Now ZN-Face starts the verification whether the graph stored under the given PIN in the database fits to the acquired image. The final decision 'yes' or 'no' is based on the significance conditions mentioned above under step (3). This does not require to search the whole database, but only the stored graphs to the given PIN and a fixed number of reference graphs have to be searched.

It has to be mentioned that even with the user's cooperation during image acquisition, there is considerable variation in the images with respect to head posture, size and position. This is due to the user-dependence of the automated image acquisition. Most of the variation can be handled successfully by the robustness of the elastic graph matching. Also, variations like different facial expressions or wearing glasses are handled very well by the algorithm. To scope with the remaining cases, the system offers the possibility to store more than one image for a given person ("teach-in", see below).

Adding new persons to the database is made very simple for an authorized system administrator by using the graphical user interface (GUI) under Windows. An image of the new user is acquired in the same way as described above, and the system automatically locates the face and extracts the new graph (Fig. 3). In addition, the GUI offers a facility to browse through the verification protocol (Fig. 4). This control panel also offers the possibility of "teach-in": If an image acquired during verification is rejected although it shows the correct person (perhaps because stored and acquired image differ too strongly in head posture, thus leading to a low significance), the authorized system administrator has the option to add the picture into the person's record in the database. The system can handle an arbitrary number of images in a persons's record, although normally 1-3 images are sufficient for reliable verification.

\footnotetext{
${ }^{1}$ Windows is a registered trademark of Microsoft
} 


\section{Results}

Nearly all papers on face recognition algorithms usually report only on the recognition rate achieved by the algorithm, i. e. how successfully a person is accepted whose image is in the database. But this is only one side of the coin: Often even more important for biometric devices is the question, how successfully persons are rejected whose image is not in the database. Biometric research names errors of the former kind as False Rejection Rate (FRR) while errors of the latter kind contribute to the False Acceptance Rate (FAR). Of course, there is a trade-off between both types of errors and consequently it is much more difficult to build a system with a high recognition rate and a low FAR than a system with a high recognition rate alone.

The system ZN-Face offers the possibility to balance optimally the trade-off between FAR and FRR. 'Optimal' is defined here as the simultaneous minimization of FAR and FRR. In the verification, the actual acceptance value is compared against an acceptance threshold. If needed, the user has the possibility to shift the acceptance threshold, e. g. in in the direction of lower FAR, usually at the expense of a somewhat larger FRR.

We conducted two experiments with a database of more than 130 persons: In the first one (Fig. 5A), the database contained one image per person. For each person, the verification was tested with a different image $\mathcal{I}$ of the same person. Unsuccessful verifications (acceptance value too low) contribute to the FRR in Fig. 5A. Then, the person was removed from the database and it was tested, whether the image $\mathcal{I}$ was now rejected, as it should. False acceptances contribute to the FAR. As can be seen from Fig. 5A, the combined minimum of FAR and FRR is achieved at a level of about 3.5\%. In Fig. 5B the same experiment is repeated, but now with 2 images per person in the database. In this case, comparing with a person's third image $\mathcal{I}$ usually yields higher acceptance values. Here, it is possible to shift the acceptance threshold in such a way, that FAR and FRR simultaneously drop to zero. (This is of course only true for the specific database, more comprehensive tests with larger data material have to be undertaken to estimate with higher accuracy, how low FAR and FRR actually are).

\section{Conclusion and outlook}

Elastic Graph Matching has proven to be a powerful algorithm for the recognition of human faces, especially because it achieves at the same time a reliable rejection of unknown faces. This makes it an appropriate tool for a biometric access control device which has been presented in this work. In addition to the results presented in the preceding section, the system has also been tested recently 'live' on several exhibitions (VISION'94 and SECURITY'94) where it operated successfully under real-world conditions with 'untrained' visitors. The fast identification of faces on standard hardware opens new application possibilities on the biometry market. The ZN Bochum GmbH has sold more 
than 100 licences to customers of various fields (among them the Kremlin in

Moscow). The ZN Bochum GmbH uses distributors proven in the field of access control and hold the leading market position in this field.

\section{References}

[1] M. Lades, J. Vorbrüggen, J. Buhmann, J. Lange, C.v.d. Malsburg, R.P. Würtz, and W. Konen. Distortion invariant object recognition in the dynamic link architecture. IEEE Transaction on Computers, 42:300-311, 1993.

[2] V. Bruce and M. Burton. Processing Images of Faces. ABLEX PublishingCorporation, Norwood, NJ, 1992.

[3] T. Kanade. Picture Processing System by Computer Complex and Recognition of Human Faces. Unpublished Ph.D. thesis, Dept. of Information Science, Kyoto Univ., 1973.

[4] R. J. Baron. Mechanisms of human facial recognition. International Journal of Man Machine Studies, 15:137-178, 1981.

[5] R. Brunelli and Tomaso Poggio. Face recognition: Features versus templates. Technical Report TR 9110-04, Istituto per la Ricerca Scientifica e Tecnologica, October 1992.

[6] J. M. Gilbert and Woodward Yang. A real-time face recognition system using custom VLSI hardware. In Proc. of Computer Architectures for Machine Perception Workshop, December 1993.

[7] M. Kirby and L. Sirovich. Application of the Karhunen-Loeve procedure for characterization of human faces. IEEE Transactions on Pattern Analysis and Machine Intelligence, 12(1):103-108, January 1990.

[8] M. Turk and A. Pentland. Face recognition using eigenfaces. In IEEE Proc. of CVPR, pages 586-591, Maui, Hawaii, June 1991.

[9] T. Kohonen, E. Oja, A. Kortekangas, and K. Mäkisara. In Proc. Intl. Conf. on Cybernetics and Scociety, Washington D.C., 1977.

[10] G. Cottrell, P. Munro, and D. Zipser. Learning internal representations of grey scale images: An example of extensional programming. In Proc. Ninth Annual Cognitive Science Society Conference, Seattle, WA, 1987.

[11] B. A. Golomb, D. T. Lawrence, and T. J. Sejnowski. SEXNET: A neural network identifies sex from human faces. In D. S. Touretzky and R. Lippman, editors, Advances in Neural Information Processing Systems 3. Morgan Kaufmann, San Mateo, 1991. 
[12] M. Bichsel and P. Seitz. Der elektronische Pförtner: Automatisches Erkennen und Identifizieren von menschlichen Gesichtern. In R.E. Grosskopf, editor, Mustererkennung 1990, 12. DAGM-Symposium. Springer, 1990. 

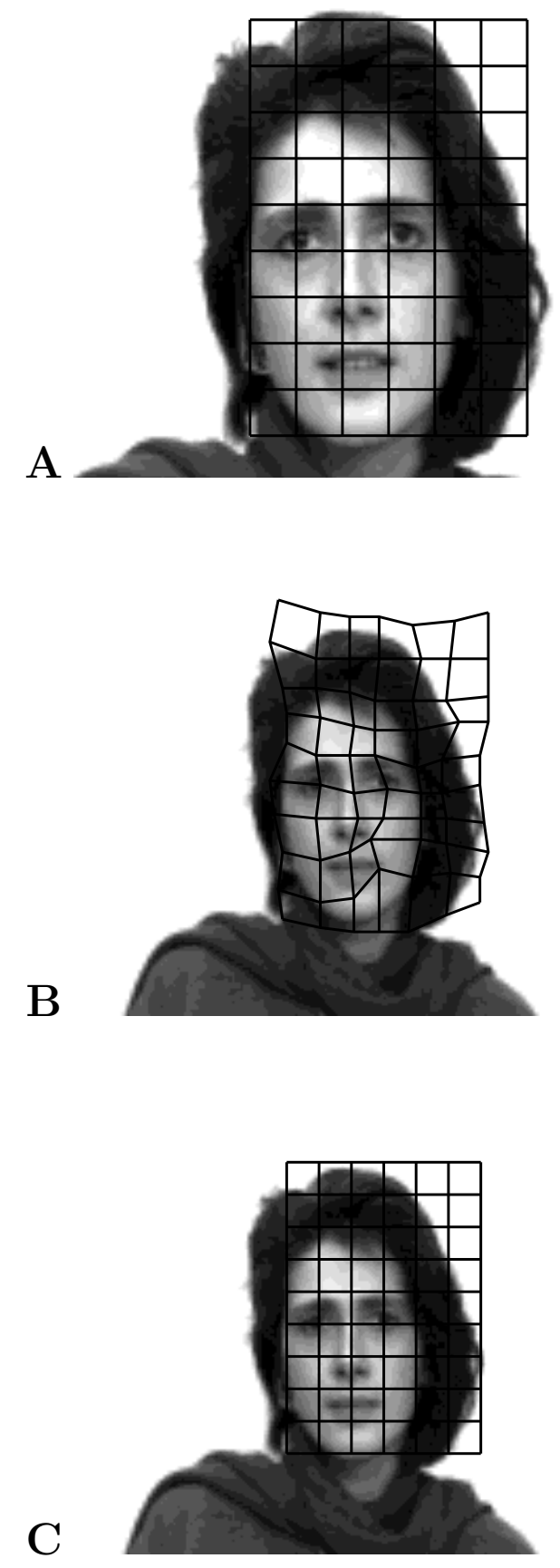

Figure 1: Data representation in $Z N$-Face. Such graphs can be shifted or scaled (C) efficiently in the image domain. 


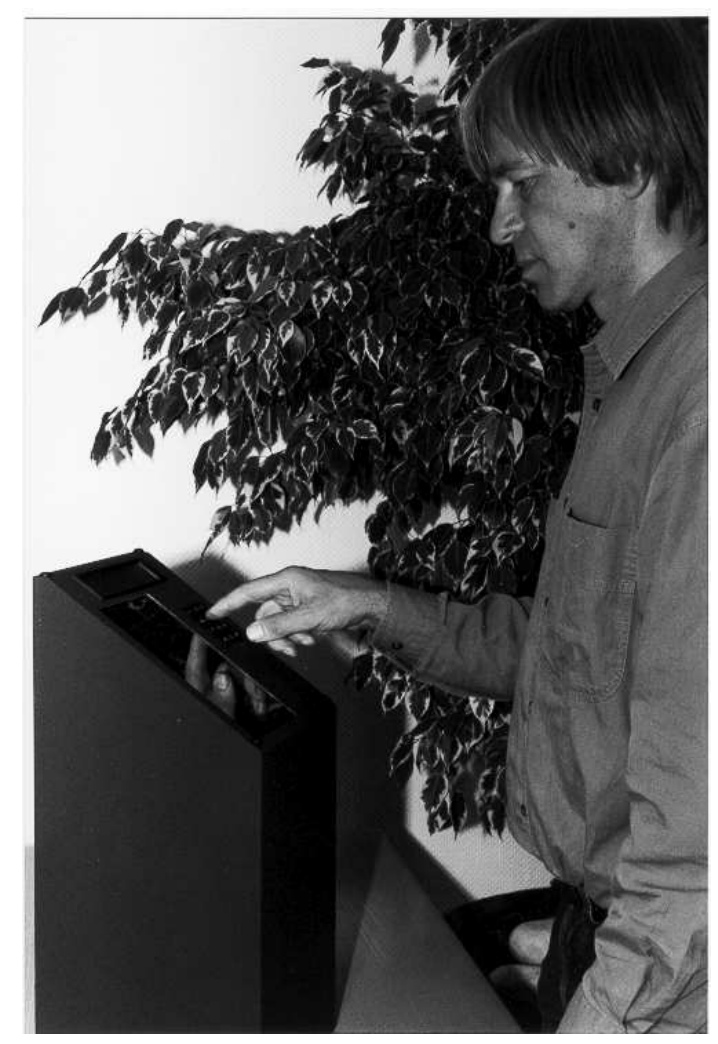

Figure 2: The access control system $Z N$-Face in a prototype realization, consisting of camera (behind semi-permeable mirror), LC-display and PIN pad.

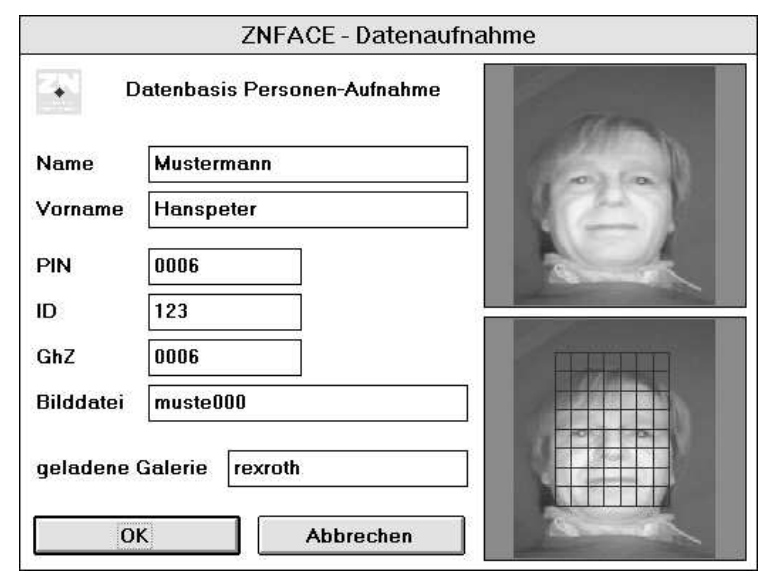

Figure 3: GUI for adding new persons to the database. The system automatically locates and extracts the new graph as shown in the lower image. 


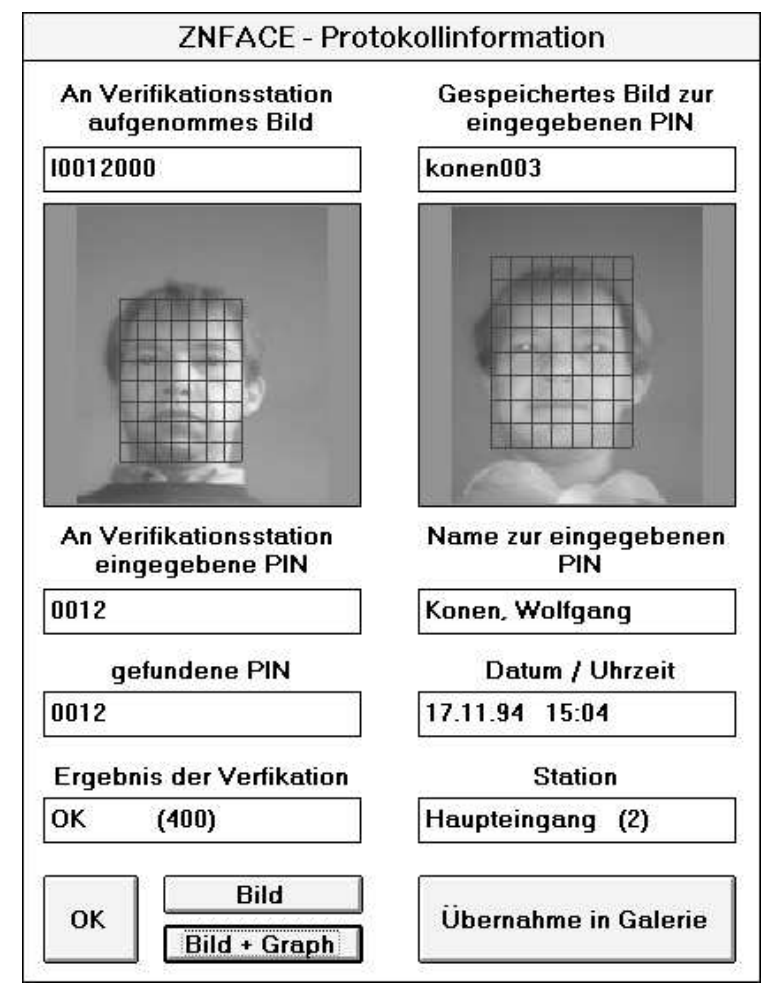

Figure 4: Protocol of the verification: On the left hand side the actually acquired image is shown, on the right hand side the best matching image from the database (to the given PIN). 


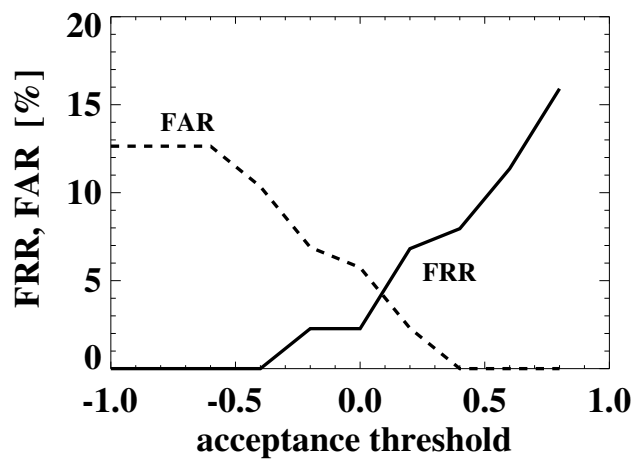

A

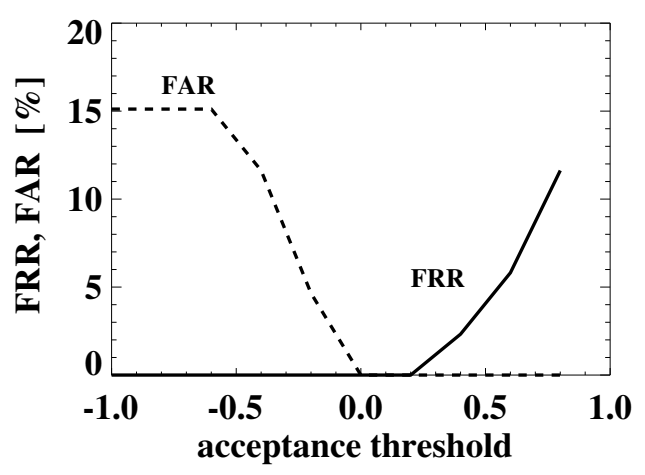

B

Figure 5: False rejection rate (FRR) and false acceptance rate (FAR) for recognition from a database of 130 persons as a function of the adjustable acceptance threshold. The threshold value 0 corresponds to the threshold learned by the neural net as optimal discrimination. The database contains one image per person in (A) and two images per person in (B). 